\title{
Nonlinear Optics of Photons and Atoms
}

\author{
Cao Long Van ${ }^{1}$, Dinh Xuan Khoa ${ }^{2}$, Marek Trippenbach ${ }^{3}$ \\ ${ }^{1}$ Institute of Physics, University of Zielona Góra \\ ul. Prof. Szafrana 4a, 65-516 Zielona Góra, Poland \\ e-mail:vanlongcao@yahoo.com \\ ${ }^{2}$ Vinh University, 182 Le Duan str., Vinh, Nghe An, Vietnam \\ ${ }^{3}$ Department of Physics, Warsaw University \\ ul. Hoża 69, 00-681 Warsaw, Poland
}

(Received: 29 June 2010; accepted: 13 September 2010)

\begin{abstract}
In this presentation we intend to focus on the exchange of experience between nonlinear optics (optical pulse and beam propagation in nonlinear media) and atom optics (dynamics of coherent waves generated from Bose-Einstein condensates). A common ground here is the nonlinear Schrödinger equation, which with the proper substitution of variables describes both types of phenomena. In nonlinear optics it is a light propagation equation that relates the signal at the end of the nonlinear crystal to the signal at the input face of the medium. In Bose-Einstein condensate dynamics it is the called the Gross-Pitaevskii equation. We will discuss various types of phenomena which have realisations both in the nonlinear optics and atom optics. We will concentrate our consideration on soliton formation, which is a challenge to fundamental and applied research in these two domains of physics.
\end{abstract}

Key words: soliton; nonlinear optics, atom optics; Gross-Pitaevskii equation

Starting from Maxwell's equations we obtain the wave equation for propagating electric field $\vec{E}(\vec{x}, t)$

$$
\begin{aligned}
& \left(\nabla_{\perp}^{2}+\frac{\partial^{2}}{\partial z^{2}}+\frac{1}{c^{2}} \frac{\partial^{2}}{\partial t^{2}}\right) \vec{E}(\vec{x}, t)= \\
& =\frac{2 \pi}{c^{2}} \frac{\partial^{2}}{\partial t^{2}}\left(\vec{P}^{L}(\vec{x}, t)+\vec{P}^{N L}(\vec{x}, t)\right),
\end{aligned}
$$

where $z$ is the direction of propagation, $\nabla_{\perp}^{2}$ is the transverse part of the laplacian, while $P^{L}$ and $P^{N L}$ are linear and nonlinear polarization vectors, respectively. In literature there is no systematic way of deriving the propagation equation for arbitrary orders of dispersion, diffraction and nonlinearities in an arbitrary medium. In [1] we have presented a method for derivation of this propagation equation in the nonlinear differential form. In a special case we introduced this equation systematically in [2].

It is well known that a great number of physical processes involved in a given nonlinear problem can be understood in terms of the formation of spatial, temporal or spatiotemporal localized structures or solitons. In our recent paper we concentrate on these subjects and review some of our latest results. The study of these localized waves is strongly complicated by the fact that the nonlinear partial differential equations (PDEs) of the given system are usually not integrable. By investigating integrability of a nonlinear PDE, one gains an important insight into the structure of the equation and the nature of its solutions. With the exception of some analytical solutions obtained by well-known methods (inverse scattering method, Hirota's method) [5] the solitary wave solutions have to be determined numerically [6]. One of the most effective methods is the Fourier Split-Step method which is used in studying dynamics of short-pulse splitting in dispersive nonlinear media [3, 4]. In some cases we should use an analytical and numerical methods simultaneously [7].

As is has been recognized before $[8,9]$ the search for spatiotemporal solitons in optical media, alias "light bullets" (LBs) [10], is a challenge to fundamental and applied research in nonlinear optics (see [11-13]). Stationary solutions for LBs can be found from the cubic $\left(\chi^{(3)}\right)$ multidimensional nonlinear Schrödinger (NLS) equation [10] which is a well-known asymptotic model governing the slow evolution of the local amplitude of the electromagnetic field [5]. However, they are unstable against spatiotemporal collapse [14]. The problem may be avoided by introducing milder nonlinearities, such as saturable [15], cubic-quintic [16], or quadratic $\left(\chi^{(2)}\right)[11,12,17]$.

Despite considerable progress in theoretical studies, three-dimensional (3D) LBs in a bulk medium have not 
been observed in an experiment yet. The only successful experimental finding reported thus far was a stable quasi2D spatiotemporal soliton in $\chi^{(2)}$ crystals [12]. The tiltedwavefront technique [18] has been used in that paper to introduce strong artificial group-velocity dispersion (GVD). This precluded achieving self-confinement in one transverse direction. On the other hand, it was predicted [17] that a spatial cylindrical soliton might be stabilized in a bulk medium composed of layers with alternating signs of the Kerr coefficient.

In the $2 \mathrm{D}$ case, we proposed a scheme for stabilizing spatiotemporal (STS) solitons in Kerr media with a layered structure. We consider an experimentally realistic possibility, viz., periodic reversal of the GVD sign, which resembles known dispersion-management (DM) schemes in fiber optics. First, we have developed the variational approximation (VA) based on the Gaussian ansatz for 2D STS. In the 2D case, simulations of the resulting systems of coupled variational equations reveal well-defined stability regions in the relevant parameter space. In [9] we verified these results by direct simulations by Split-Step Fourier Method mentioned above using the MATLAP program. We again obtained a new stable object in the form of a periodically oscillating bound state of two subpulses. As a byproduct we concluded that the program in MATLAP was twice faster than the program used before in FORTRAN.

In our further work [19], we proposed a possibility to stabilize spatiotemporal solitons ("light bullets") in threedimensional self-focusing Kerr media by means of dispersion management (DM), which means that the local group-velocity dispersion coefficient alternates between positive and negative values along the propagation direction, $z$. The model is based on a scalar equation of the NLS type, which can be derived in the paraxial approximation for the slowly varying amplitude of a linearly polarized electromagnetic wave. We showed previously that the DM alone could stabilize solitons in 2D (planar) waveguides, but in the bulk (3D) DM medium the "bullets" were unstable. In [19] we demonstrated that complete stabilization could be provided if the longitudinal DM was combined with periodic modulation of the refractive index in one transverse direction $(y)$, with a period much larger than the carrier wavelength. A stability area for the light bullets was identified in the model parameter space. Its salient features are a necessary minimum strength of the transverse modulation of the refractive index, and minimum and maximum values $E_{\min \text { max }}$ of the soliton energy. The former feature can be accurately predicted in an analytical form from the evolution equation for the width of the soliton in the $y$-direction. Also, similar to the case of DM soliton in fibers, we find that the stability area extends into a region of normal average dispersion [20]. On the other hand, the existence of $E_{\max }$ can be understood similarly to the way it was done in the 2D counterpart of the present model (the strong transverse lattice can squeeze the system to a nearly $2 \mathrm{D}$ shape).

The model opens a way to address further issues, such as collisions between the LBs, and the existence and stability of solitons with different symmetries (for instance, LBs which are odd in the longitudinal and (or) transverse directions).

As recognized in our previous paper [21], by analogy between the propagation equation in the Kerr medium and the Gross-Pitajevski equation for Bose Einstien condensates (BECs), we could transfer the results from nonlinear optics to atom optics and vice versa [7, 22]. A similar stabilization mechanism was then predicted for 2D solitons in Bose-Einstein condensates (BECs), with the coefficient in front of the cubic nonlinear term subjected to a periodic modulation in time via the Feshbach resonance in an external ac magnetic field [23-25]. However, no stable 3D soliton could be predicted in either realization of this setting (optical or BEC).

In [26] we investigated the stability properties of breather soliton in a three-dimensional Bose-Einstein Condensate with "Feshbach Resonance Management" of the scattering length and confined only by a one-dimensional optical lattice. We compared the region of stability in parameter space obtained from a fully 3D analysis with those from a quasi two-dimensional treatment. For moderate confinement we discovered a new island of stability in the $3 \mathrm{D}$ case, not present in the quasi $2 \mathrm{D}$ treatment. Stable solutions from this region have nontrivial dynamics in the lattice direction, hence they describe fully 3D breather solitons. We demonstrated these solutions in direct numerical simulations and more importantly, suggest a way of creating robust 3D solitons in experiments in a BoseEinstein Condensate in a one-dimensional lattice.

\section{References}

[1] Cao Long Van, M. Trippenbach, New Optical Solitary waves in the higher order nonlinear Schrödinger Equation. Scientific Biuletin 126, Technical University of Zielona Gora, Poland 2001, p. 19 (Cao Long Van ed.).

[2] V. Cao Long, H. Nguyen Viet, M. Trippenbach, K. Dinh Xuan, Propagation Technique for Ultrashort Pulses I. Computational Methods in Science and Technology 14 (1), 5 (2008).

[3] V. Cao Long, H. Nguyen Viet, M. Trippenbach, K. Dinh Xuan, Propagation Technique for Ultrashort Pulses II. Computational Methods in Science and Technology 14 (1), 13 (2008). 
[4] V. Cao Long, H. Nguyen Viet, M. Trippenbach, K. Dinh Xuan, Propagation Technique for Ultrashort Pulses III. Computational Methods in Science and Technology 14(1), 21 (2008).

[5] Cao Long Van, P.P. Goldstein, A concise Course in nonlinear Partial Differential Equations, University of Zielona Gora (2008).

[6] A.V. Buryak, Yu. S. Kivshar, Phys. Lett. A197, 407 (1995).

[7] M. Trippenbach, M. Matuszeski, E. Infreld, Cao Long Van, R.S. Tasgal, J.B. Band, Enhancement of third harmonic generation by wave vector mismatch to counter phasemodulation. Optics Communications 229, 391 (2004).

[8] M. Matuszewski, M. Trippenbach, B.A. Malomed, E. Infeld, A.A. Skorupki, Two dimensional dispersion managed light bullets in a Kerr medium. Phys. Rev. E70, 016603 (2004).

[9] Nguyen Viet Hung, Cao Long Van, M. Trippenbach, Stability of two dimensional dispersion managed light bullet in a Kerr medium. Communications in Physics 17, suppl. 51 (2007).

[10] Y. Silberberg, Collapse of optical pulses. Opt. Lett. 15, $1282(1990)$

[11] K. Hayata, M. Koshiba, Multidimensional solitons in quadratic nonlinear media. Phys. Rev. Lett. 71, 3275 (1993).

[12] X. Liu, L.J. Qian, F.W. Wise, Generation of Optical Spatiotemporal Solitons. Phys. Rev. Lett. 82, 4631 (1999).

[13] B.A. Malomed, D. Mihalache, F. Wise, L. Torner, Spatiotemporal optical solitons. J. Opt. B: Quant. Semicl. Opt. 7, R53 (2205).

[14] L. Berge', Wave collapse in physics: principles and applications to light and plasma waves. Phys. Rev. 303, 259 (1998).

[15] D.E. Edmundson, R.H. Enns, Roust bistable light bullets. Opt. Lett. 17, 586 (1992).

[16] D. Mihalache at el., Stable spinning optical solitons in three dimensions. Phys. Rev. Lett. 88, 073902, 1-4 (2002).

[17] I. Towers, B.A Malomed, Stable (2+1)-dimensional solitons in a layered medium with sign-alternating Kerr nonlinearlity. J. Opt. Soc. Am. B19, 537 (2002).
[18] P. Di Trapani et al., Observation of Temporal Solitons in Second - Harmonic Generation with Tilted Pulses. Phys. Rev. Lett. 81, 570 (1998).

[19] M. Trippenbach et al., Two-and three-dimensional light bullets in a Kerr medium with dispersion management. (to be published in Phys. Rev. A).

[20] J.H.B. Nijhof, N.J. Doran, W. Forysiak, F.M. Knox, Stable soliton like propagation in dispersion managed systems with net anomalous, zero and normal dispersion. Electron. Lett. 33, 1726 (1997).

[21] Dinh Xuan Khoa, Cao Long Van, M. Trippenbach, Nonlinear Optics of Photons and Atoms. Proceeding of National Conference on Theoretical Physics, Hue, Vietnam, 15-18 August 2005.

[22] M. Trippenbach, Cao Long Van, Vu Ngoc Sau, Nguyen Huy Bang, Bragg scattering of Bose-Einstein Condensates an Imaginary Time Method. Proceeding of National Conference on Theoretical Physics, Sam son, Vietnam, 12-14 August 2003.

[23] H. Staito, M. Ueda, Dynamically Stabilized Bright Solitons in a two Dimensional Bose Einstein. Phys. Rev. Lett. 90, 040403 (2003).

[24] F. Kh. Abdulaev, J.G. Caputo. R.A. Kraenkel, B.A. Malomed, Controlling collapse in Bose-Einstein condensates by temporal modulation of the scattering length. Phys. Rev. A67, 013605 (2003).

[25] G.D. Montesinos. V.M. Perez-Grarcia, P.J. Torres, Stabilization of soliton of the multidimensional nonlinear Schrödinger equation: Matter-Wave breathers. Physic D191, 193 (2004).

[26] M. Trippenbach, M. Matuszewski, Cao Long Van, E. Infeld, B.A. Malomed, Fully three-dimensional breather solitons can be created using Feshbach resonance. Communications in Physics 17, suppl. 111 (2007).

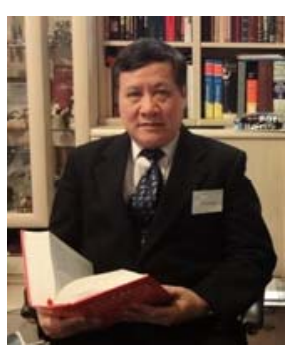

Cao Long VAN was born in 1952 in Hanoi, Vietnam. He graduated from Warsaw University in 1976 with an MSc degree in Theoretical Physics. His master thesis was entitled "Callan-Symanzik Equation and Transition Probabilities" and was written under the supervision of Prof. Iwo Białynicki-Birula. In 1979, he defended his $\mathrm{PhD}$ dissertation entitled "Phase Representation in Quantum Optics", also supervised by Prof. I. BiałynickiBirula. He habilitated in 1987 in the Center of Theoretical Physics, Polish Academy of Sciences in Warsaw with a thesis entitled "Noises in Quantum Optics". He is recently extraordinary professor of University of Zielona Góra, Vice-Dean of Faculty of Physics and Astronomy. His research interests concern several topics in Quantum and Nonlinear Optics.

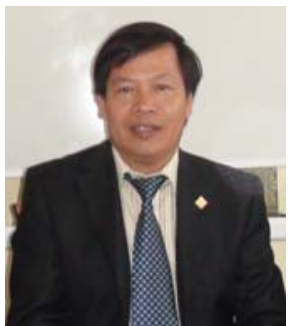

DiNH XUAN KHOA raduated from Vinh University, Vietnam, with a major in physics in 1981. He completed a PhD course in Quantum Optics in 1996. His doctoral thesis entitled "Generative Kinetics of Dye Lasers" was supervised by Prof. Cao Long Van and Prof. Dao Xuan Hoi. His field of interests covers a large variety of topics in Quantum and Nonlinear Optics. He is exceptionally interested in Soliton Theory and research concerning long-distance optical communication systems. Recently, Prof. Dinh Xuan Khoa is recently rector of Vinh University. 


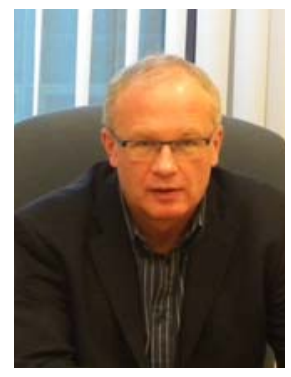

MareK TRIPPEnBaCh, PhD, DSc, full professor of physics, is a graduate of Warsaw University. His field of interests covers various topics of Quantum and Nonlinear Optics. His recent research is focused on solitons in such nonlinear systems as Optical Nonlinear Media and Bose-Einstein condensates. He was a co-author of the well-known experiment which was carried out by the group led by a Nobel Prize laureate from 1997: William Phillips. In this experiment, the Four-Wave Mixing of Condensates was observed. This phenomenon is considered to be the first observable effect of Nonlinear Atom Optics. During his stay in JILA (Boulder, Colorado), Marek Trippenbach worked with yet another laureate of the Nobel Prize (from 2001): Carl Wieman who was among the first to experimentally discover the Bose-Einstein Condensate. Nowadays, he is working at the Institute of Theoretical Physics, Warsaw University. He is a fellow in the British Institute of Physics. He is recently Vice-Dean of Departament of Physics, Warsaw University. 